\title{
Metastasis of Rhabdomyosarcoma to the Male Breast: a Case Report with Magnetic Resonance Imaging Findings
}

Investigative Magnetic Resonance Imaging

\section{Case Report}

Received: October 10, 2018 Revised: November 5, 2018 Accepted: December 26, 2018

\section{Correspondence to:}

Bong Joo Kang, M.D., Ph.D. Department of Radiology, Seoul St. Mary's Hospital, College of Medicine, The Catholic University of Korea, 222, Banpo-daero, Seocho-gu, Seoul 06591, Korea. Tel. +82-2-2258-6253

Fax. +82-2-599-6771

E-mail: gmlionmain@gmail.com

This is an Open Access article distributed under the terms of the Creative Commons Attribution Non-Commercial License (http://creativecommons.org/licenses/ by-nc/4.0/) which permits unrestricted non-commercial use, distribution, and reproduction in any medium, provided the original work is properly cited.

Copyright (C) 2019 Korean Society of Magnetic Resonance in Medicine (KSMRM)

\section{Myeongjong Kim ${ }^{1}$, Bong Joo Kang ${ }^{2}$, Ga Eun Park ${ }^{2}$, Sung Hun Kim², Jeongmin Lee $^{2}$, Ahwon Lee ${ }^{3}$}

${ }^{1}$ Department of Radiology, St. Vincent's Hospital, The Catholic University of Korea, Suwon, Korea ${ }^{2}$ Department of Radiology, Seoul St. Mary's Hospital, The Catholic University of Korea, Seoul, Korea

${ }^{3}$ Department of Pathology, Seoul St. Mary's Hospital, The Catholic University of Korea, Seoul, Korea

Metastasis of rhabdomysarcoma to the breast is a very rare manifestation in adult males. Herein, we report a case of metastasis from embryonal rhabdomyosarcoma in the left hypothenar muscle that presented as a breast mass in a 38-year-old man, who four months later expired because of multiple bone metastases related to pancytopenia. We describe the various imaging findings, including mammograms, ultrasonography, computerized tomography (CT), positron emission tomographycomputed tomography (PET-CT), and magnetic resonance imaging (MRI) of this rare disease. The various imaging findings of this lesion could be helpful for future diagnosis of male breast lesions.

Keywords: Rhabdomyosarcoma; Adult; Breast; Metastasis; Metastatic rhabdomyosarcoma

\section{INTRODUCTION}

Rhabdomyosarcoma is exceedingly infrequent in adults. Soft tissue sarcomas constitute less than $1 \%$ of all adult malignancies, and rhabdomyosarcoma accounts for $3 \%$ of all soft tissue sarcomas (1). Nonetheless, it is a common childhood cancer, constituting more than $50 \%$ of all soft tissue sarcomas. The head-neck region is the most common primary site (1). And the most common metastatic sites from primary rhabdomyosarcoma are lung, bone, bone marrow, and liver. Breast metastases of rhabdomyosarcoma are rare and have mainly been reported in adolescent females (24). Breast metastases from extramammary malignancies are very rare, particularly in men. To our knowledge, there has only been one reported case of rhabdomyosarcoma metastasis to a male breast (4). In this study, we present a case of a 38-year-old man with breast metastasis from rhabdomyosarcoma. We describe the imaging findings, including mammograms, ultrasonography, computerized tomography (CT), positronemission tomography-computed tomography (PET-CT), and magnetic resonance imaging (MRI) of this rare disease. 


\section{CASE REPORT}

A 38-year-old male patient was diagnosed with embryonal rhabdomyosarcoma in the left hypothenar muscle and underwent neoadjuvant chemotherapy. After two cycles of chemotherapy (vincristine, doxorubicin, and cyclophosphamide), the lesion decreased in size, and he underwent resection of the primary rhabdomyosarcoma, followed by local radiation therapy. After six months, metastasis to the axillary lymph node was confirmed, and he underwent resection of the lymph nodes followed by local radiation and adjuvant chemotherapy (vincristine, ifosfamide, and etoposide). After five months, there were palpable masses in both breasts. Breast ultrasonography was suggestive of gynecomastia (Fig. 1). After two months, the size of the palpable mass in the left breast increased. On follow-up ultrasonography, a $4 \times 1.7 \mathrm{~cm}$ irregular mass with a heterogeneous internal echo pattern and internal vascularity was detected in the left subareolar-tocentral region (Fig. 2a, b). On a mammogram, an irregular,
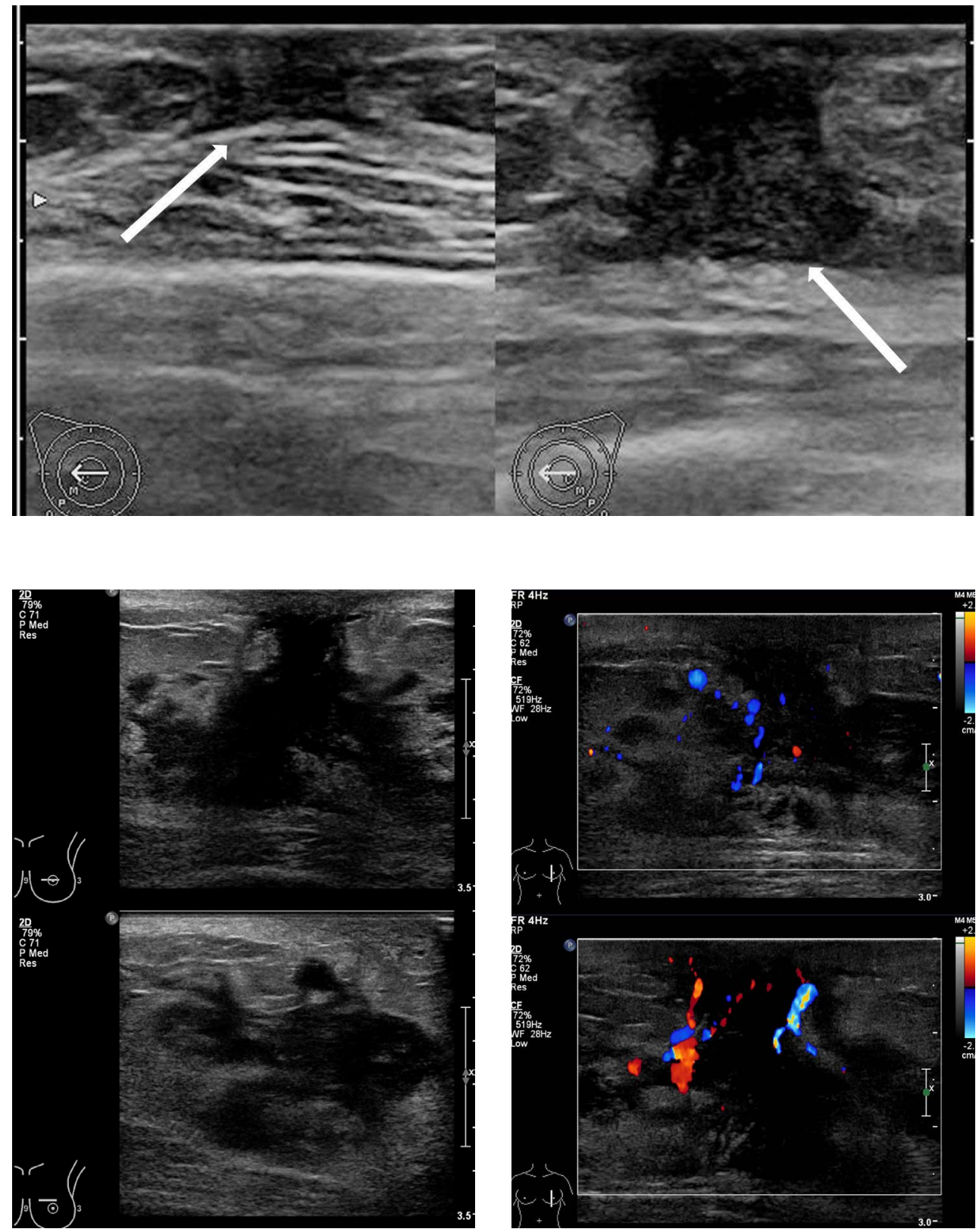

a

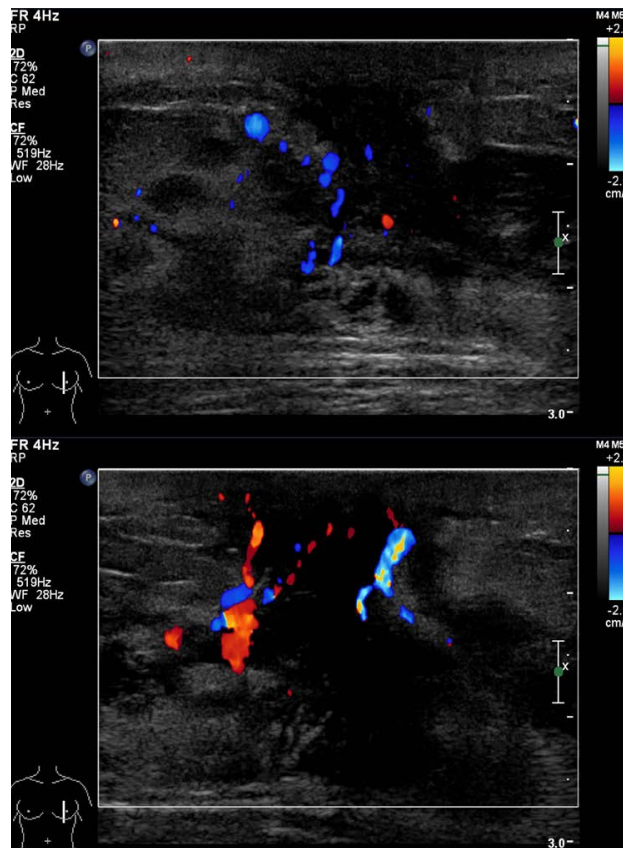

b
Fig. 1. Ultrasonographic image reveals hypoechoic fibroglandular tissue in both subareolar spaces, more on the left, suggesting gynecomastia (arrows).

Fig. 2. Follow-up ultrasonographic image (a) and color Doppler scan (b) reveals an irregular mass of about $4 x$ $1.7 \mathrm{~cm}$ with a heterogeneous internal echo pattern in the left subareolar-tocentral region with internal vascularity, suggesting metastasis of a known rhabdomyosarcoma. 
circumscribed, hyperdense mass of about $5.9 \mathrm{~cm}$ was found in the left subareolar-to-central region, in agreement with the ultrasonography (Fig. 3). Contrast-enhanced CT showed a lobulated heterogenous enhancing mass in the left breast (Fig. 4), and PET-CT showed a hypermetabolic mass (maximum standardized uptake value, SUVmax: 11.8) in the left breast (Fig. 5).

Contrast-enhanced MRI (Verio, Siemens Healthineers, USA) for bone and soft tissue mass (left upper extremity) showed a mass of about $5.1 \times 2.1 \times 5.7 \mathrm{~cm}$ with isointense signal intensity (SI), compared to the muscle on a T1weighted image (WI) (Fig. 6a) and high SI on T2WI (Fig. $6 b)$. The signal of the mass was not suppressed on fatsuppressed T2WI (Fig. 6c), and showed heterogeneous enhancement on contrast-enhanced T1WI (Fig. 6d), with high SI on high $b$ value diffusion-weighted images $(b=800$
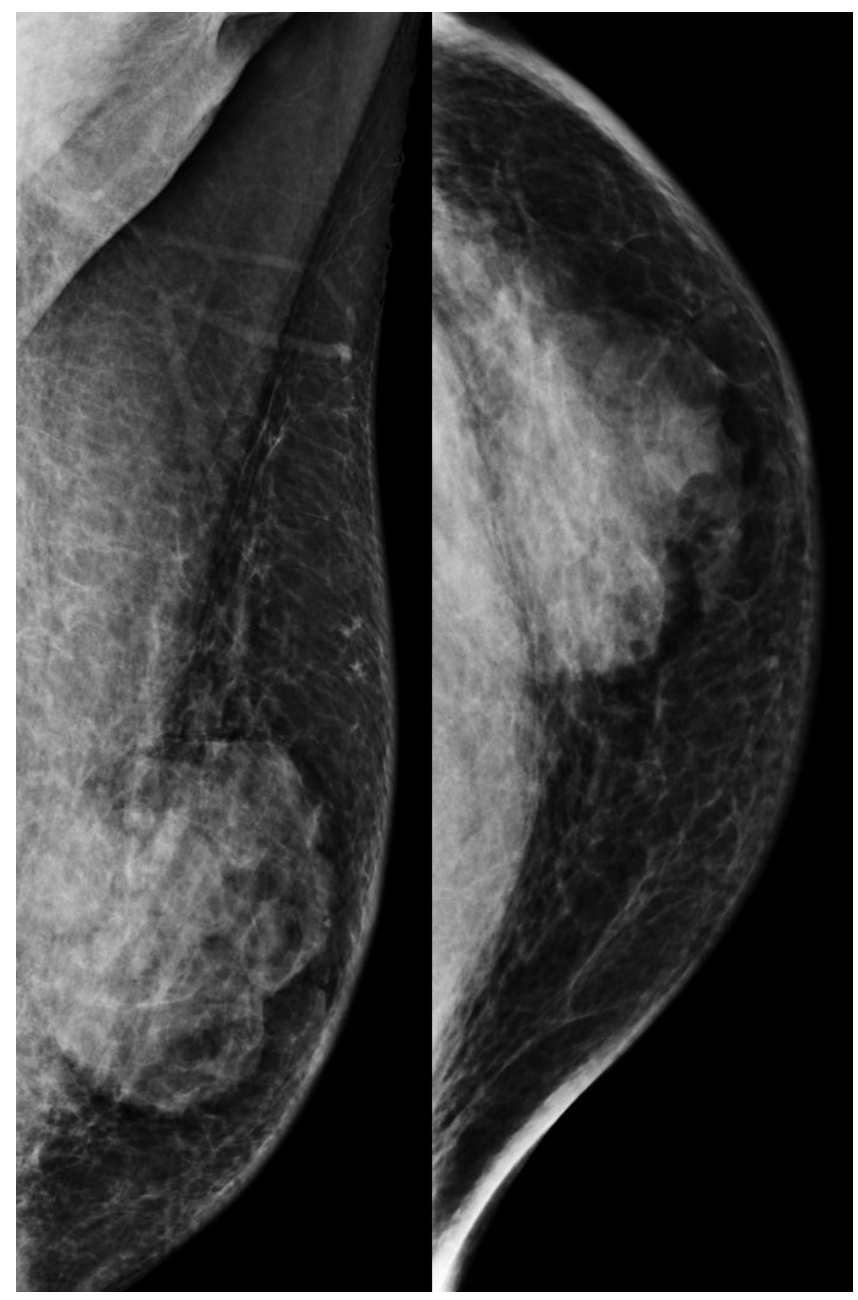

Fig. 3. Mammograms of the left breast reveal an irregular, circumscribed, hyperdense mass of about $5.9 \mathrm{~cm}$ in the left subareolar-to-central region. $\mathrm{s} / \mathrm{mm}^{2}$ ) and a definitive low apparent diffusion coefficient (ADC) value (mean: $0.65 \times 10^{-3} \mathrm{~s} / \mathrm{mm}^{2}$ ) in the subareolar space of the left breast (Fig. 6f).

The patient subsequently underwent wide mass excision, which found a gross specimen, a well-demarcated, pale brown tumor mass of about $5 \times 5 \times 3 \mathrm{~cm}$ with hemorrhagic foci (Fig. 7). Microscopic examination revealed small roundto-oval cells with eccentrically located vesicular nuclei and clear-to-eosinophilic cytoplasm that had diffusely

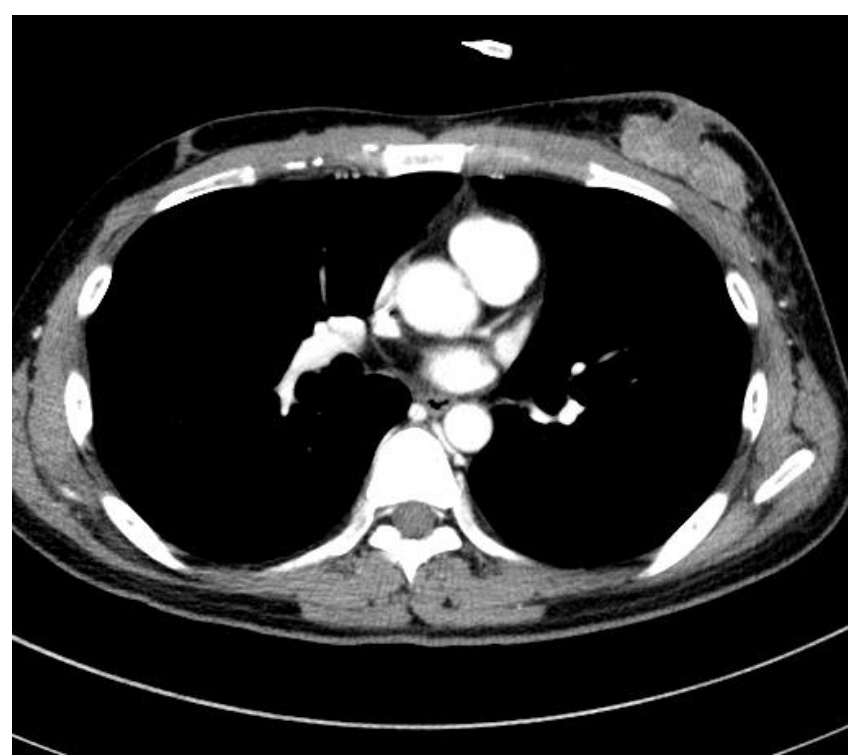

Fig. 4. Contrast-enhanced CT image reveals a lobulated, heterogeneous enhancing mass of about $5.3 \mathrm{~cm}$ in the left subareolar-to-central region.

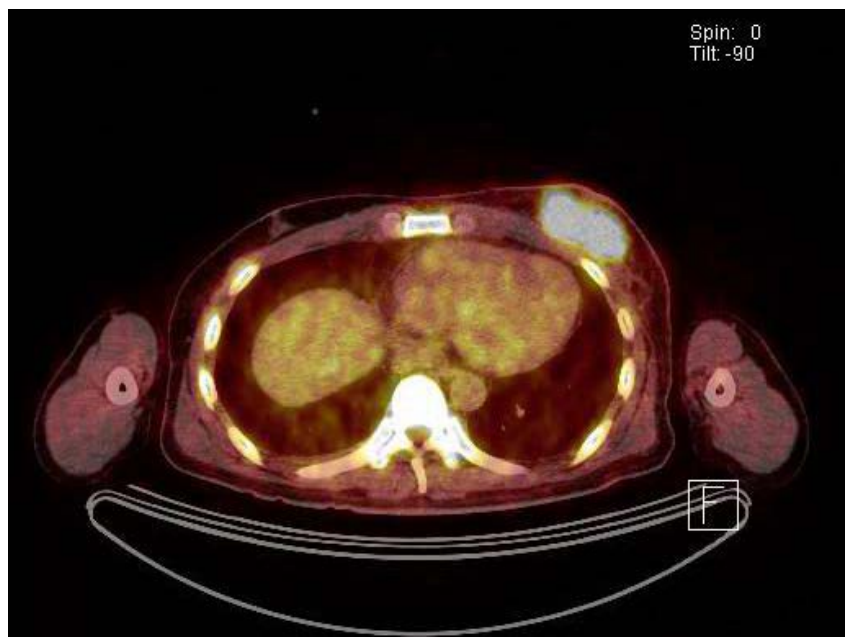

Fig. 5. Positron-emission tomography-computed tomography (PET-CT) shows a hypermetabolic mass in the left breast (SUVmax: 11.8). 


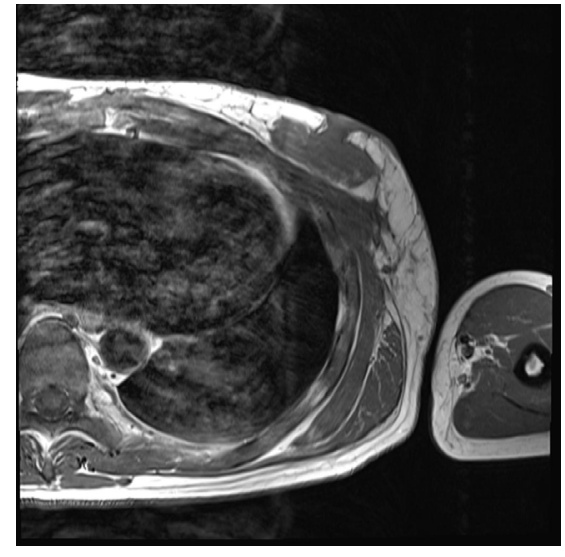

a

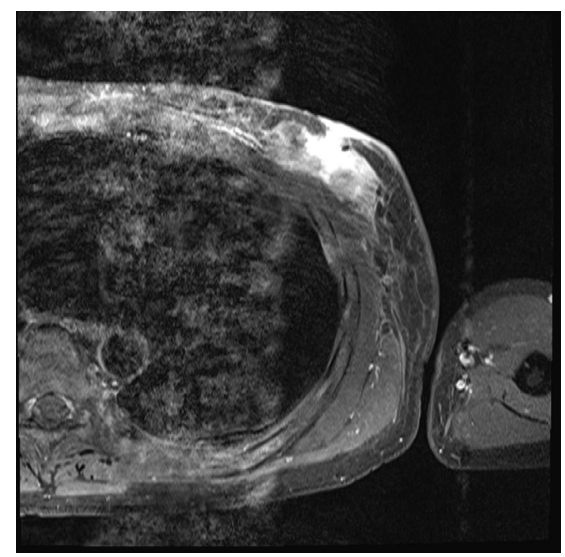

d

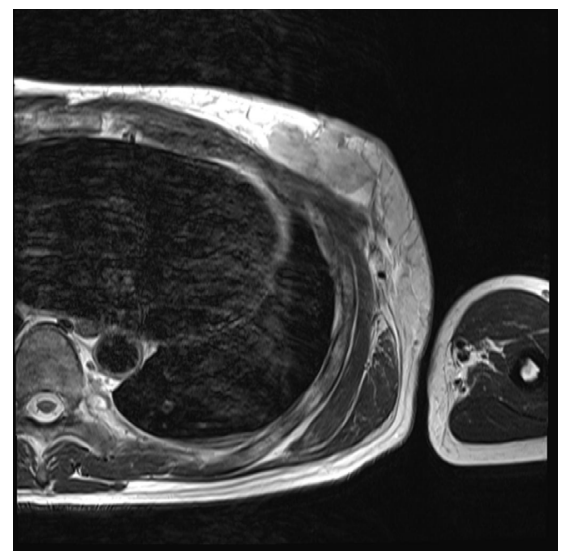

b

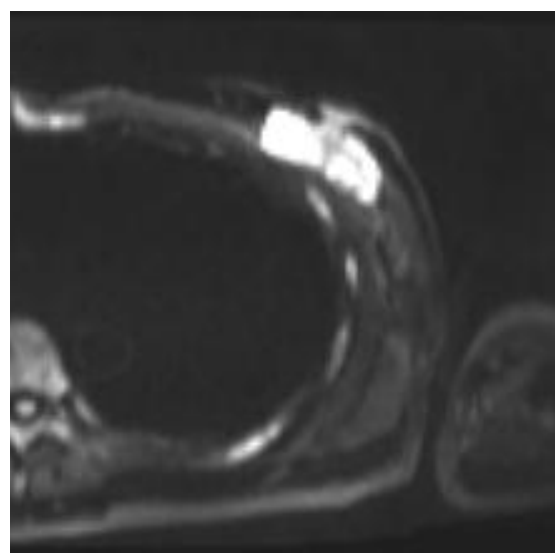

e

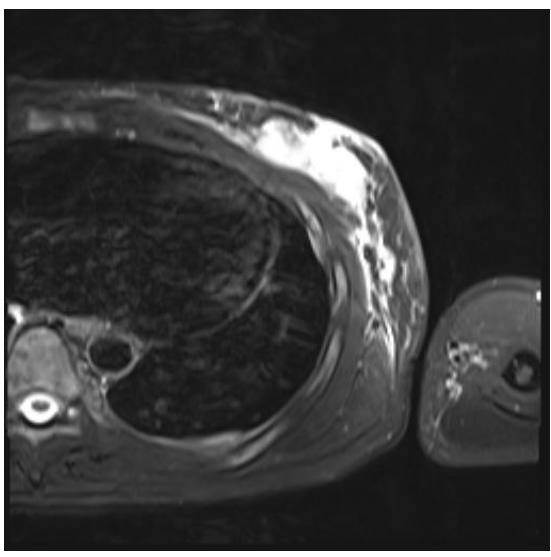

c

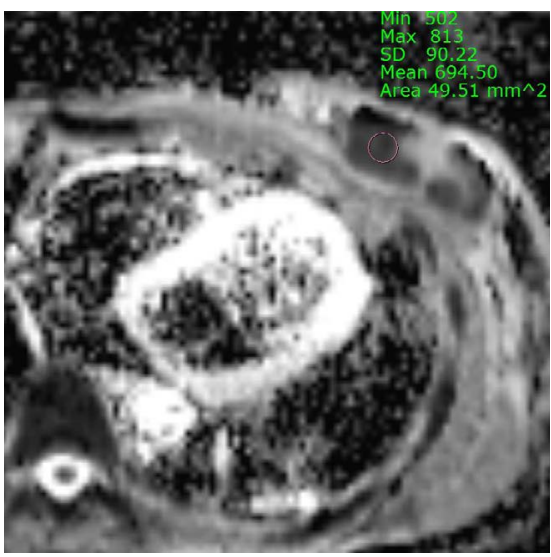

f

Fig. 6. T1- (a), T2- (b), fat-suppressed T2- (c), and 2 minute delayed gadolinium-enhanced T1-weighted (d) MR images. An axial MRI showed isointense signal intensity, compared to the muscle in contrast T1 weighted image (WI), with high SI on T2Wl. The signal of the mass was not suppressed on fat-suppressed T2WI and showed heterogeneous enhancement on contrast-enhanced T1WI with high SI on a high b value diffusion-weighted image $\left(b=800 \mathrm{~s} / \mathrm{mm}^{2}\right)$ (e), with a definitive low apparent diffusion coefficient (mean $\left.0.69 \times 10^{-3} \mathrm{~s} / \mathrm{mm}^{2}\right)(f)$ in the left breast.

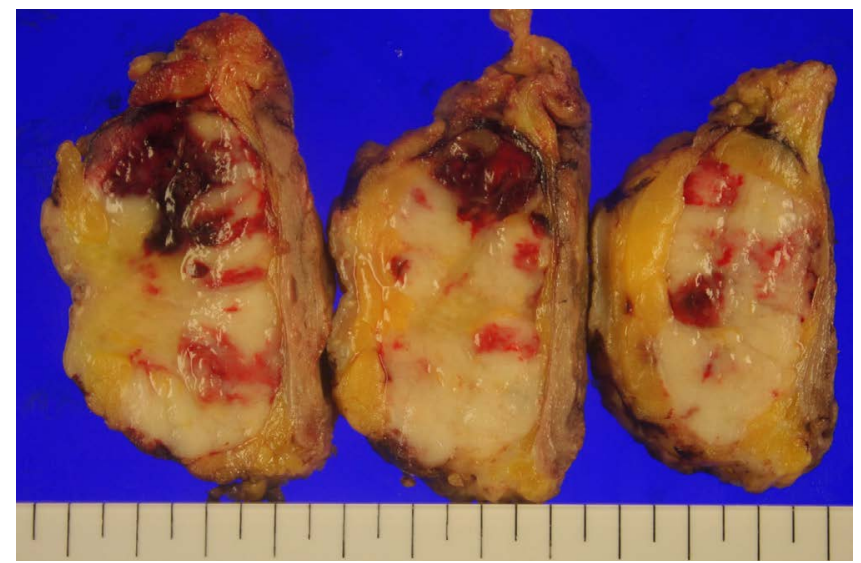

Fig. 7. A well-demarcated tumor mass, $5.0 \times 5.0 \times 3.0 \mathrm{~cm}$, pale brown, solid cut surfaces with hemorrhagic foci. infiltrated the breast parenchyma (Fig. 8a, b). Tumor cells stained positive for myogenic marker (MyoD1) (Fig. 8c) and were confirmed to be metastatic rhabdomyosarcoma. Additionally, MRI and PET-CT revealed multiple thoracic, lumbar spine, and sacral metastases, as well as metastases in both pelvic bones and both femurs. Lung metastases were detected on chest CT. After four months, the patient expired because of cardiac and pulmonary dysfunction due to probable disseminated intravascular coagulation (DIC) from pancytopenia-related multiple bone metastases. 


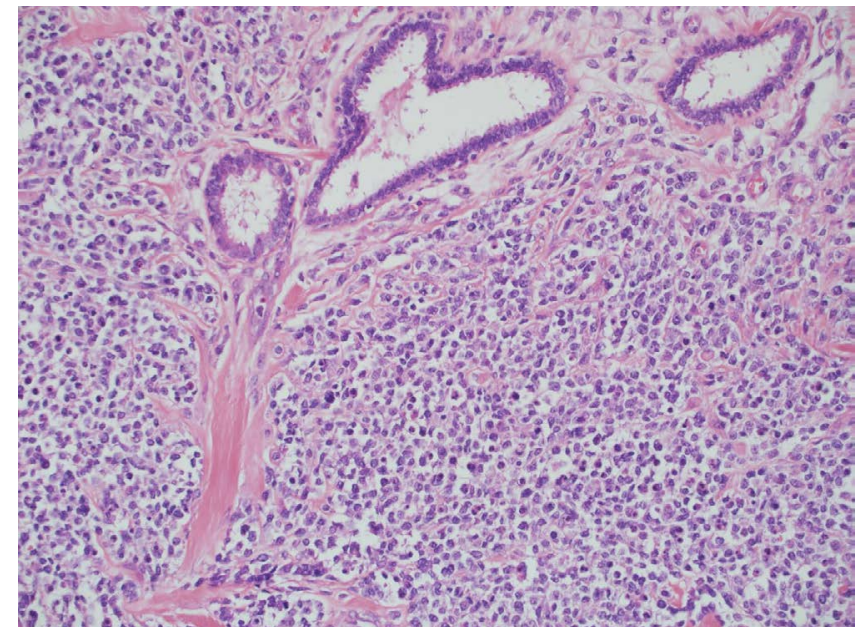

a

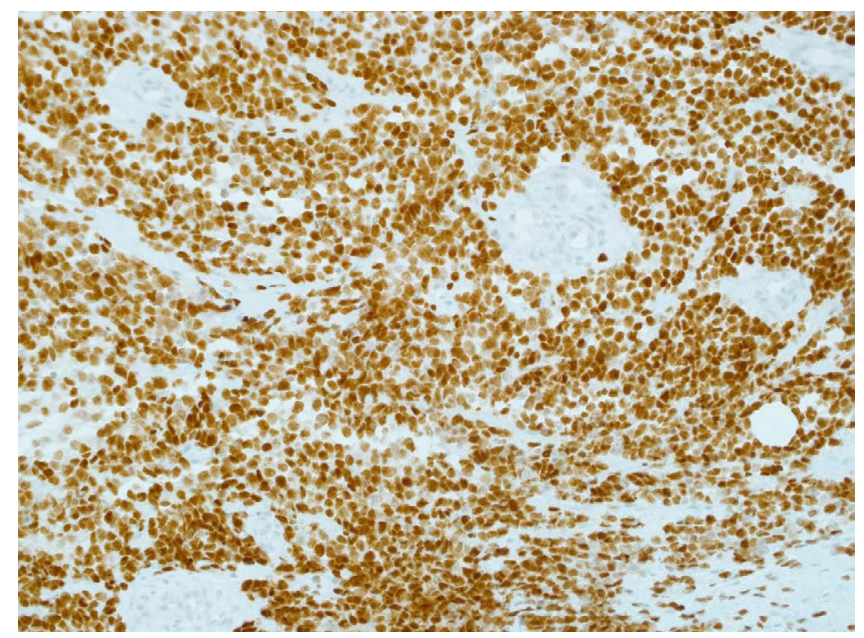

c

\section{DISCUSSION}

Rhabdomyosarcoma is a rare malignancy and metastases to the breast are uncommon lesions. Autopsy incidence accounts for $0.5-6.6 \%$ of all breast malignancies. However, the clinically-observed rate of metastases ranges from 0.6 to $1.3 \%(2,5,6)$, perhaps because the breast contains large areas of fibrous tissue with a relatively poor blood supply (6).

In our case, three unusual features were present. First, this was a male patient with metastatic rhabdomyosarcoma to the breast. Female patients are affected five to six times more frequently than male patients (7).

Second, he was a 38-year-old adult. To our knowledge, there is no reported care of metastasis of rhabdomyosarcoma to the male breast. Seventy cases of children and adolescents below 19 years of age with breast metastasis of

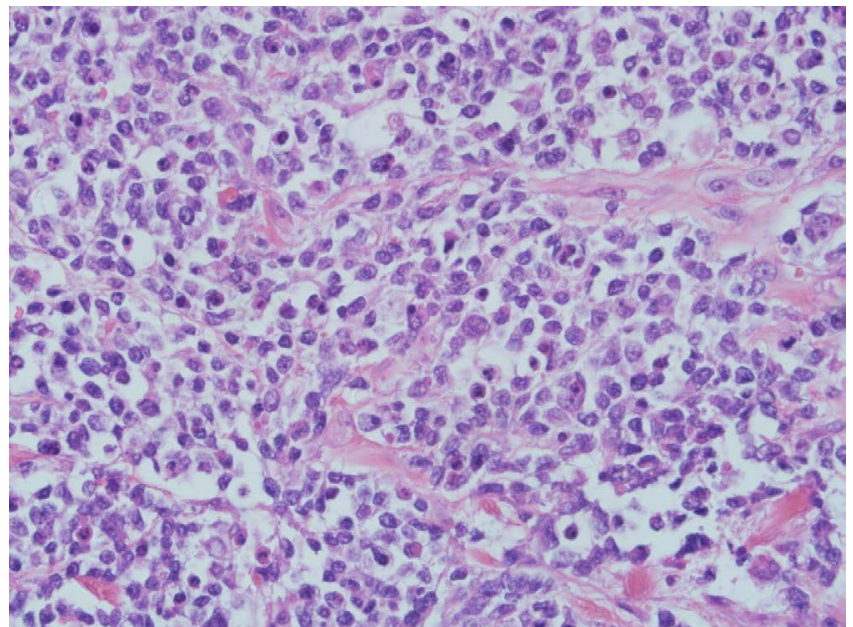

b

Fig. 8. Metastatic rhabdomyosarcoma was confirmed by pathology. (a) Tumor cells are small, round to oval cells, diffusely infiltrating the breast parenchyma $(\times 200$, Hematoxylin \& Eosin [H\&E] staining). (b) Tumor cells show eccentrically located vesicular nuclei and clear-toeosinophilic cytoplasm ( $\times 400, H \& E$ staining). (c) Tumor cells are diffusely positive for MyoD1 (x 200 MyoD1 immunohistochemical staining).

rhabdomyosarcoma were reported from 1980 to 2014 (4). Both primary and breast metastases of rhabdomyosarcoma have mainly been reported in adolescent girls (2-4). Since most of these patients were younger women, it is plausible that the increasing vascularity and rapidly growing mammary tissue during puberty may be related to preferential metastasis to the breast (4). In our case, the patient was diagnosed with gynecomastia and breasts were developing, as in a pubertal girl. The developing breast is at a higher risk for being involved in metastasis (4).

Last, this patient's pathology report confirmed embryonal rhabdomyosarcoma. Most rhabdomyosarcoma cases reported with breast metastasis had an alveolar histology (4). In one analysis of 19 cases of rhabdomyosarcoma with initial metastases to the breasts, all of rhabdomyosarcoma were of the alveolar subtype (8). Howarth et al. (9) followed-up on 108 
consecutively-diagnosed patients with rhabdomyosarcoma and found that $6 \%$ of them subsequently developed metastatic tumors of the breast. They noted that $85 \%$ had alveolar histology.

There is no previous report of breast metastasis from embryonal-type rhabdomyosarcoma to the male breast. Our patient was a young adult, and the histology was of the embryonal type. To the best of our knowledge, this is the first case describing an extremity metastasizing embryonal rhabdomyosarcoma to an adult male breast. The imaging findings of this lesion in mammograms, ultrasonography, $\mathrm{CT}, \mathrm{PET}-\mathrm{CT}$, and even MRI could be helpful for future diagnosis of male breast lesions. Our results suggest that metastasis should be considered in adult males with a breast mass, particularly those with a personal history of rhabdomyosarcoma.

\section{REFERENCES}

1. Ferrari $A$, Dileo $P$, Casanova $M$, et al. Rhabdomyosarcoma in adults. A retrospective analysis of 171 patients treated at a single institution. Cancer 2003;98:571-580

2. Birjawi GA, Haddad MC, Tawil AN, Khoury NJ. Metastatic rhabdomyosarcoma to the breast. Eur Radiol 2001;11:555558
3. Hays DM, Donaldson SS, Shimada H, et al. Primary and metastatic rhabdomyosarcoma in the breast: neoplasms of adolescent females, a report from the Intergroup Rhabdomyosarcoma Study. Med Pediatr Oncol 1997;29:181-189

4. Kebudi R, Koc BS, Gorgun O, Celik A, Kebudi A, Darendeliler E. Breast metastases in children and adolescents with rhabdomyosarcoma: a large single-institution experience and literature review. J Pediatr Hematol Oncol 2017;39:6771

5. Noguera J, Martinez-Miravete $P$, Idoate $F$, et al. Metastases to the breast: a review of 33 cases. Australas Radiol 2007;51:133-138

6. Surov A, Fiedler E, Holzhausen HJ, Ruschke K, Schmoll HJ, Spielmann RP. Metastases to the breast from nonmammary malignancies: primary tumors, prevalence, clinical signs, and radiological features. Acad Radiol 2011;18:565-574

7. Toombs BD, Kalisher L. Metastatic disease to the breast: clinical, pathologic, and radiographic features. AJR Am J Roentgenol 1977;129:673-676

8. Nguyen HV, Aminololama-Shakeri S, Zhang Y. Initial presentation and recurrence of metastatic rhabdomyosarcoma as breast mass. Radiol Case Rep 2013;8:855

9. Howarth CB, Caces JN, Pratt CB. Breast metastases in children with rhabdomyosarcoma. Cancer 1980;46:25202524 